\title{
Incidence and predictive value of postoperative Troponin elevation in high-risk patients undergoing Major thoracic surgery
}

\author{
Coronado Silva CC, Morales P, Cano E, Vázquez C, González-Tallada A, de Nadal M. \\ Department of Anaesthesiology \& Intensive Care. \\ Hospital Universitari Vall d'Hebron. Universitat Autònoma de Barcelona. Barcelona, Spain
}

Background and Goal of Study: Cardiac troponins are considered reliable biomarkers of myocardial injury in patients who undergo thoracotomy and lung surgery (1).Troponin elevation has been associated with intrapericardial resections and pneumonectomies, but the incidence reported and its predictive value largely varies upon series $(2,3)$. The peak of troponin measurement during the first 3 days after noncardiac surgery was an independent predictor of 30-day mortality (4). The aims of this study were to determine the incidence of postoperative troponin elevation after major thoracic surgery in high-risk patients, and its correlation with the extension of the procedure and 30-day mortality.

Patients and methods: As part of routine postoperative care for high cardiac risk patients ( $\geq 65$ years or $<65$ years with known cardiovascular pathology), we measured serum highsensitivity Troponin I (Tnl) levels (Siemens ADVIA Centaur) during the first two postoperative days in 117 consecutive patients undergoing major thoracic surgery. Patients were divided in two groups upon Tnl elevation. The Tnl cut off used to define troponin elevation was the 99th percentile provided by our laboratory $(\mathrm{Tnl} \geq 0.04 \mathrm{ng} / \mathrm{ml}$ ). Baseline clinical characteristics, perioperative parameters, and 30-day mortality were assessed in both groups.

Results and discussion: From January 2014 to October 2015, 117 high-risk patients underwent postoperative Tnl monitoring after major thoracic surgery, of which $35(25.7 \%)$ had a peak $\mathrm{Tnl} \geq 0.04 \mathrm{ng} / \mathrm{ml}$ on postoperative day 1 or 2 . $\mathrm{Tnl}$ elevation was more frequent after pneumonectomy and lobectomy (P-value chi-squared 0.046) (Table1). Overall 30day mortality was of $3 / 117(2.6 \%)$ and no difference was found between patients with or without postoperative troponin elevation (P-value 0.154) (Table 2).

Active smoking was an independent factor for postoperative troponin elevation (P-value 0.028). No differences were found in age, sex or baseline clinical characteristics (including history of coronary artery disease) neither in perioperative parameters.

Conclusions: In our study, $25.7 \%$ of high-risk patients undergoing major thoracic surgery showed postoperative troponin elevation, being more frequent in more extensive procedures. 30-day mortality showed no difference between patients with or without troponin elevation. This two results suggest that troponin elevation in thoracic surgery may be related to other mechanisms other than myocardial ischemia. Further studies are needed to confirm this issue.
TABLE 1: Lung resection, Tnl elevation and 30-day mortality

\begin{tabular}{|c|c|c|c|c|}
\hline \multirow{2}{*}{$\begin{array}{c}\mathrm{N}(\%) \\
{[95 \% \mathrm{Cl}]}\end{array}$} & \multicolumn{2}{|c|}{ TROPONIN I $\geq 0.04 \mathrm{ng} / \mathrm{ml}$} & \multirow{2}{*}{ Total } & \multirow{2}{*}{$\begin{array}{c}\text { P-value } \\
\text { Chi-squared }\end{array}$} \\
\hline & Yes & No & & \\
\hline Lobectomy & $\begin{array}{c}26(37.14 \%) \\
{[27.17 \% ; 49.52 \%]}\end{array}$ & $\begin{array}{c}44(62.86 \%) \\
{[51.93 \% ; 74.11 \%]}\end{array}$ & $\begin{array}{c}70(59.83 \%) \\
{[50.36 \% ; 68.78 \%]}\end{array}$ & \\
\hline Pneumonectomy & $\begin{array}{c}3(60 \%) \\
{[28.36 \% ; 94,73 \%]}\end{array}$ & $\begin{array}{c}2(40 \%) \\
{[14.66 \% ; 85.34 \%]}\end{array}$ & $\begin{array}{c}5(4.27 \%) \\
{[1.4 \% ;} \\
9.69 \%]\end{array}$ & \\
\hline Wedge resection & $\begin{array}{c}5(19.23 \%) \\
{[8.97 \% ; 39.35 \%]}\end{array}$ & $\begin{array}{c}21(80.77 \%) \\
{[65.13 \% ; 93.45 \%]}\end{array}$ & $\begin{array}{c}26(22.22 \%) \\
{[15.06 \% ; 30.84 \%]}\end{array}$ & 0.046 \\
\hline Pleural resection & $\begin{array}{c}1(8,33 \%) \\
{[2.09 \% ; 38.48 \%]}\end{array}$ & $\begin{array}{c}11(91.67 \%) \\
{[73.54 \% ; 99.79 \%]}\end{array}$ & $\begin{array}{c}12(10.26 \%) \\
{[5.41 \% ; 17.23 \%]}\end{array}$ & \\
\hline Others & $\begin{array}{c}0(0 \%) \\
{[.63 \% ; 60.24 \%]}\end{array}$ & $\begin{array}{l}4(100 \%) \\
{[. \% ; \%]}\end{array}$ & $\begin{array}{c}4(3.42 \%) \\
{[.94 \% ; 8.52 \%]}\end{array}$ & \\
\hline 30-day mortality & $\begin{array}{c}0(0 \%) \\
{[.84 \% ; 70.76 \%]}\end{array}$ & $\begin{array}{l}3(100 \%) \\
{[. \% ; . \%]}\end{array}$ & $\begin{array}{c}3(2,56 \%) \\
{[.53 \% ; 7.31 \%]}\end{array}$ & 0.154 \\
\hline
\end{tabular}

TABLE 2. Demographic and surgical parameters

\begin{tabular}{|c|c|c|c|c|}
\hline \multirow{2}{*}{$\begin{array}{c}\mathrm{N}(\%) \\
{[95 \% \mathrm{Cl}]}\end{array}$} & \multicolumn{2}{|c|}{ TROPONIN I $\geq 0.04$ ng/ml } & \multirow{2}{*}{ Total } & \multirow[b]{2}{*}{$\begin{array}{c}\mathrm{P} \text { - value } \\
\text { Chi-squared }\end{array}$} \\
\hline & $\begin{array}{l}\text { YES } \\
n=35\end{array}$ & $\begin{array}{c}\text { NO } \\
n=85\end{array}$ & & \\
\hline Sex (Male /Female) & $\begin{array}{c}28(31 \%) / \\
7(26 \%)\end{array}$ & $\begin{array}{c}62(69 \%) / \\
20(74 \%)\end{array}$ & $\begin{array}{c}90(77 \%) / \\
27(23 \%)\end{array}$ & 0.606 \\
\hline Age $\geq 65 y r$ & $\begin{array}{c}33(29 \%) \\
{[21.62 \% ; 38.19 \%]}\end{array}$ & $\begin{array}{c}81(71 \%) \\
{[62.74 \% ; 79.16 \%]}\end{array}$ & $\begin{array}{c}114(97.44 \%) \\
{[92.69 \% ; 99.47 \%]}\end{array}$ & 0.159 \\
\hline Smoker & $\begin{array}{c}14(50 \%) \\
{[33.87 \% ; 69.37 \%]}\end{array}$ & $\begin{array}{c}14(50 \%) \\
{[33.87 \% ; 69.35 \%]}\end{array}$ & $\begin{array}{c}28(23.93 \%) \\
{[16.53 \% ; 32.7 \%]}\end{array}$ & 0.028 \\
\hline Coronary artery disease & $\begin{array}{c}5(35.71 \%) \\
{[17.66 \% ; 64.86 \%]}\end{array}$ & $\begin{array}{c}9(64.29 \%) \\
{[41.9 \% ; 87.24 \%]}\end{array}$ & $\begin{array}{c}14(11.97 \%) \\
{[6.7 \% ; 19.26 \%]}\end{array}$ & 0.613 \\
\hline $\begin{array}{c}\text { Peripheral vascular } \\
\text { disease }\end{array}$ & $\begin{array}{c}7(33.33 \%) \\
{[18.11 \% ; 64.86 \%]}\end{array}$ & $\begin{array}{c}14(66.67 \%) \\
{[47.82 \% ; 85.41 \%]}\end{array}$ & $\begin{array}{c}21(17.95 \%) \\
{[11.4 \% ; 26.12 \%]}\end{array}$ & 0.706 \\
\hline Renal chronic disease & $\begin{array}{c}2(25 \%) \\
{[8.52 \% ; 65.09 \%]}\end{array}$ & $\begin{array}{c}6(75 \%) \\
{[47.35 \% ; 96.81 \%]}\end{array}$ & $\begin{array}{c}8(6.84 \%) \\
{[3 \% ; 13.03 \%]}\end{array}$ & 0.753 \\
\hline $\begin{array}{l}\text { Intraoperative blood } \\
\text { transfusion }\end{array}$ & $\begin{array}{c}0(0 \%) \\
{[2.5 \% ; 97.5 \%]}\end{array}$ & $\begin{array}{l}1(100 \%) \\
{[. \% ; . \%]}\end{array}$ & $\begin{array}{c}1(.85 \%) \\
{[.02 \% ; 4.67 \%]}\end{array}$ & 0.512 \\
\hline
\end{tabular}

